\title{
Polymorphisms in Urea Cycle Enzyme Genes are Associated with Persistent Pulmonary Hypertension of the Newborn
}

\author{
Dinushan C Kaluarachchi, MBBS ${ }^{1}$, Caitlin J Smith, BS ${ }^{2}$, Jonathan M. Klein, MD ${ }^{1}$, Jeffrey C \\ Murray, MD'1, John M Dagle, MD, PhD¹, and Kelli K Ryckman, PhD $^{2}$ \\ ${ }^{1}$ Department of Pediatrics, University of lowa, lowa City, lowa \\ ${ }^{2}$ Department of Epidemiology, College of Public Health, University of lowa, lowa City, lowa
}

\begin{abstract}
Background-Persistent pulmonary hypertension of the newborn (PPHN) is characterized by elevated pulmonary vascular resistance (PVR). Endogenous nitric oxide is critical for regulation of PVR . Nitric oxide is generated from $\mathrm{L}$-arginine, supplied by the urea cycle (UC). We hypothesized that polymorphisms in UC enzyme genes and low concentrations of UC intermediates are associated with PPHN.
\end{abstract}

Methods-Family based candidate gene analysis to study 48 single nucleotide polymorphisms in 6 UC enzyme genes. Genotyping was done on 94 infants with PPHN and their parents. We also performed a case-control analysis of 32 cases with PPHN and 64 controls to identify the association between amino acid levels on initial newborn screening and PPHN.

Results-Three SNPs in carbamoyl phosphate synthetase 1 gene (CPS1) showed significant association with PPHN ( $\mathrm{p}=0.02)$. Tyrosine levels were significantly lower $(\mathrm{p}=0.003)$ and phenylalanine levels were significantly higher $(\mathrm{p}=0.01)$ in cases with PPHN. There was no difference in the arginine or citrulline levels between the two groups.

Conclusions-This study suggests a potential association between SNPs in the CPS1 and PPHN. Tyrosine level was significantly lower and phenylalanine level was significantly higher in cases with PPHN. These findings warrant further replication in larger cohorts of patients.

\section{Introduction}

Persistent pulmonary hypertension of the newborn (PPHN) is characterized by sustained elevation of pulmonary vascular resistance. High pulmonary vascular resistance in the setting of normal or low systemic vascular resistance leads to extrapulmonary right to left shunting across the patent ductus arteriosus and foramen ovale. This can result in life threatening hypoxemia, right ventricular failure and even death, making PPHN a serious neonatal disease. PPHN results from failure of pulmonary vascular transition to extrauterine

Corresponding Author Details: Dinushan C. Kaluarachchi MBBS, 200, Hawkins Drive, Iowa City, IA 52242, 319356 7096/ dinushankaluarachchi@uiowa.com/ Fax 3193564685.

Financial Disclosure: The authors have no financial relationships relevant to this article to disclose

Conflict of Interest: The authors have no conflicts of interest to disclose

Category of study: Translational Research 
life. It occurs mostly in term or near term infants and occurs in 2/1000 newborn infants ${ }^{1,2}$. PPHN can be idiopathic or can result secondary to neonatal pulmonary diseases such as congenital diaphragmatic hernia, pulmonary hypoplasia, respiratory distress syndrome, pneumonia and meconium aspiration syndrome.

Inhaled nitric oxide (iNO) is the mainstay of current PPHN treatment. It is a potent selective pulmonary vasodilator. Inhaled nitric oxide has decreased the need for extracorporeal membrane oxygenation (ECMO) in randomized controlled trials ${ }^{3,4}$. Endogenous nitric oxide plays a critical role in regulation of pulmonary vascular resistance and transition of pulmonary circulation at birth $^{5,6}$.

The main function of the urea cycle is to convert excess nitrogen in the form of ammonia to urea, which is excreted through the kidneys. Five key enzymes make up the urea cycle: carbamoyl-phosphate synthetase 1 (CPS1), ornithine transcarbamylase (OTC), argininosuccinate synthetase (ASS1), argininosuccinate lyase (ASL), and arginase 1 (ARG1). An additional enzyme, $\mathrm{N}$-acetylglutamate synthase (NAGS), provides CPS1 with its essential cofactor. Nitric oxide is endogenously synthesized from the precursor L-arginine by nitric oxide synthetase (NOS) ${ }^{7}$. L -arginine is an amino acid supplied by the urea cycle and is the link between the urea cycle and PPHN. Low L-arginine levels can theoretically decrease nitric oxide synthesis and lead to pulmonary hypertension.

Previous research has shown polymorphisms in urea cycle enzyme genes are associated PPHN. In one study including 31 subjects, Pearson et al. discovered that Thr1405 variant of CPS1 was associated with PPHN ${ }^{8}$. A more recent study including 36 subjects evaluated another form of pulmonary hypertension in neonates, pulmonary hypertension associated with bronchopulmonary dysplasia (BPD) and discovered an arginase 1 single nucleotide polymorphism (SNP) that may be protective against pulmonary hypertension ${ }^{9}$. Past studies have also shown that low plasma concentrations of arginine and nitric oxide metabolites are associated with PPHN $^{9}, 10$.

In the current study, we hypothesize that polymorphisms in urea cycle genes and low concentrations of specific urea cycle intermediates are associated with PPHN. We performed a family based candidate gene analysis to investigate the association between SNPs in urea cycle enzyme genes and PPHN. We also performed a case-control study to identify the association between amino acid levels and PPHN.

\section{Results}

Genotyping was completed in 94 cases and their families (total of 265 individuals). Seventy seven of these were part of a complete triad (affected neonate and both parents) and seventeen of these were part of a dyad (affected neonate and one parent). The majority of the probands were male $(61 \%)$ and Caucasian $(83 \%)$. Average gestational age was $38.3(+/-1.8)$ weeks and average birth weight was $3532 \mathrm{~g}(+/-626)$. Nineteen (20\%) of the infants were born before 37 weeks gestation. Sixty six infants $(70 \%)$ were treated with iNO and nine $(10 \%)$ of them required ECMO. A summary of demographic and clinical characteristics is provided in Table 1. 
Three SNPs in carbamoyl phosphate synthetase 1 gene (rs41272673, rs4399666, rs2287599) showed association with PPHN ( $\mathrm{p}=0.02$ ) (supplemental table). On haplotype analysis, presence of CCACTA alleles at rs2287599, rs7607412, rs7572146, rs6724941, rs3213784, and rs1047891 of $C P S 1$ was associated with PPHN ( $\mathrm{p}=0.006$ ). Also the presence of CC alleles at rs2287599 and rs7607412 of CPS1 was associated with PPHN (p=0.009). None of the SNPs in the other 5 urea cycle enzymes genes were associated with PPHN. SNPs that meet nominal significance, did not reach formal levels of significance when the conservative Bonferroni correction is applied.

Baseline characteristics of the case control study groups were similar (Table 3). Mean tyrosine level was significantly lower in the cases with PPHN compared to control group $(\mathrm{p}=0.003)$. Mean phenylalanine level was significantly higher in the cases with PPHN $(\mathrm{p}=0.01)$. There was no significant difference in the other metabolite levels between the two groups.

The relationship between urea cycle gene polymorphisms and amino acid levels were not evaluated due to the smaller size of the study group.

\section{Discussion}

PPHN is a major clinical problem in the neonatal intensive care units and can contribute significantly to morbidity and mortality in both term and near term neonates ${ }^{1,11-13}$. Various risk factors are associated with PPHN, but the exact etiology is unknown. Genetic factors may increase susceptibility to PPHN ${ }^{14-15}$.

Variants in urea cycle enzyme genes have been associated with various cardiopulmonary diseases in children and adults. These cardiopulmonary diseases include asthma and bronchodilator response ${ }^{16,17}$, myocardial infarction ${ }^{18}$, hypertension ${ }^{19}$, increased pulmonary artery pressure following surgical repair of congenital heart defects ${ }^{20}$, hepatic veno occlusive disease following bone marrow transplantation ${ }^{21,22}$ and $\mathrm{PPHN}^{8}$.

In the current study, we performed a family-based candidate gene analysis including 94 neonates with PPHN and their parents. The family-based study design identifies risk alleles by examining nonrandom allele transmission from parents to offspring, avoiding issues associated with population stratification. We also studied 48 SNPs across all six urea cycle enzyme genes, a significantly higher number of markers than previous studies on this topic.

We identified 3 new SNPs in carbamoyl phosphate synthetase I gene (rs41272673, rs4399666, rs2287599) associated with PPHN. SNPs rs41272673 and rs4399666 are intronic SNPs and rs2287599 is a synonymous mutation. None of the SNPs have been previous reported to be associated with disease states. The previously reported CPS1 T1405N polymorphism (rs1047891) by Pearson et al. ${ }^{8}$ was not associated with PPHN in our cohort $(\mathrm{p}=0.25)$. Haplotype analysis identified the presence of CCACTA alleles at rs2287599, rs7607412, rs7572146, rs6724941, rs3213784, and rs1047891 of CPS1 was associated with PPHN ( $\mathrm{p}=0.006$ ). This effect was mostly mediated by the presence of CC alleles at rs2287599 and rs7607412. SNPs that met nominal significance, did not reach formal levels 
of significance when the conservative Bonferroni correction is applied ( $\mathrm{p}<0.001)$, but given the exploratory nature of this study less stringent values are also of interest.

Carbamyl phosphate synthetase 1 (CPSI) is the rate-limiting enzyme located inside the mitochondrion that catalyzes the first committed step of the urea cycle. As it's the rate limiting enzyme of the urea cycle, changes in CPS1 carry greater functional effect than the other enzymes in the pathway. The CPSI1 gene is located on human chromosome 2q35, consists of 38 exons and 37 introns that span more than 120 kilobases $^{23}$. Genetic variants in CPS1 gene can affect the functional efficiency of the CPS1 enzyme especially under environmental stress conditions. The CPS1 T1405N polymorphism(rs1047891) has been studied widely and is associated with clinical outcomes under various environmental stresses such as increased pulmonary artery pressure following surgical repair of congenital heart defects ${ }^{21}$, hepatic veno occlusive disease following bone marrow transplantation ${ }^{21}$ and $\mathrm{PPHN}^{8}$. These associations are referred to as environmentally determined genetic expression (EDGE) effects ${ }^{22}$. We can speculate that the 3 additional SNPs identified by our current study affect the functional efficiency of CPS1 limiting the substrate availability for nitric oxide. Increased demand for nitric oxide associated with neonatal stress following child birth, in the setting of decreased functional efficiency of CPSI might bring out the PPHN phenotype.

Several studies have detected lower urea cycle intermediate levels including arginine and citrulline in the neonates with $\mathrm{PPHN}^{8,10}$. In the current study, we did not find a difference in arginine or citrulline levels between the two groups, but the tyrosine level was significantly lower in the cases with PPHN. Nitration of tyrosine by nitrogen species such as peroxynitrite can result in nitrotyrosine ${ }^{24,25}$. Nitrotyrosine, a biomarker of oxidative stress has been found in elevated levels in patients with bronchopulmonary dysplasia ${ }^{26}$. Patients with PPHN are managed with mechanical ventilation, high levels of inspired oxygen and inhaled nitric oxide. Hyperoxic ventilation can lead to formation of reactive oxygen species and formation of nitrotyrosine, which could in turn reflect as low tyrosine levels.

We also found that levels of phenylalanine were higher in cases with PPHN. Higher phenylalanine levels have been observed in preterm newborns with respiratory distress syndrome ${ }^{27}$. Marginally higher levels of phenylalanine are also seen in preterm newborns with patent ductus arteriosus ${ }^{27}$. Elevated phenylalanine levels could be due to impaired phenylalanine hydroxylase (PAH) activity. Depletion of 5,6,7,8-tetrahydrobiopterin, a cofactor for PAH can impair the PAH activity ${ }^{28}$. Oxidative stress due to formation of reactive oxygen species can deplete 5,6,7,8-tetrahydrobiopterin resulting in impaired PAH activity. This can explain the high phenylalanine concentrations in PPHN. Impaired PAH activity can also explain the low tyrosine levels, as PAH converts phenylalanine to tyrosine.

Our study has several limitations. Our sample size is relatively smaller, even though we used a larger sample size compared to prior studies of a similar nature. Also the majority of the families were Caucasian, so the results may not be reflective of other ethnicities. The retrospective nature of the study is another limitation. Our use of a family-based study design is a strength allowing for the examination of transmission of alleles from the parent to affected offspring an approach that had not been previously used to investigate urea cycle 
enzyme genes. Our study also included a greater number of SNPs compared to previous studies.

In conclusion, this study suggests a potential association between SNPs in the CPS1 gene and PPHN. We identified three previously unreported SNPs in CPS1 gene associated with PPHN. These polymorphisms might affect the functional efficiency of CPS1 leading to PPHN. There was no difference in the arginine and citrulline levels between cases with PPHN and unaffected controls. Tyrosine levels were significantly lower and phenylalanine levels were significantly higher in cases with PPHN. These findings need to be replicated in a large cohort of patients.

\section{Methods}

The study consisted of two phases. In the first phase genotyping of single nucleotide polymorphisms in urea cycle enzyme genes was performed. The second phase was a casecontrol analysis to identify the association between amino acid levels and PPHN.

\section{Study Population}

The study population for genotyping consisted of 94 neonates diagnosed with and treated for PPHN at the University of Iowa Children's Hospital between 1993 and 2010 and their families (Table 1). Inclusion criteria were neonates with hypoxemic respiratory failure with the clinical diagnosis of pulmonary hypertension. Exclusion criteria were gestational age $<35$ weeks, multiple major congenital anomalies, congenital diaphragmatic hernia, cyanotic heart disease, and/or the inability to obtain a DNA sample from the neonate and at least one parent.

Neonates with hypoxic respiratory failure were diagnosed with PPHN by the medical team using echocardiography, preductal/ postductal oxygen saturation difference $>10 \%$, and/or a clinical response to inhaled nitric oxide. Echocardiographic findings consistent with PPHN included elevated pulmonary artery pressure as compared with systemic pressure, right-toleft or bidirectional patent ductus arteriosus shunting and right-to-left or bidirectional shunting through the patent foramen ovale.

Samples were obtained from the DNA repository at the University of Iowa. DNA was extracted from venous blood, cord blood, buccal swabs or saliva. Demographic and clinical information were collected through medical chart abstraction.

All participants or their guardians provided signed informed consent for study enrollment in accordance with the protocols approved by University of Iowa Institutional Review Board (IRB 200307031).

\section{SNP Genotyping}

We performed a family based candidate gene analysis to study $48 \mathrm{SNPs}$ (Table2) in 6 urea cycle enzyme genes (CPS1, NAGS, ASS, ASL, ARG1, OTC). SNPs were selected according to known functional impact and according to a catalog of variants of urea cycle enzyme genes by Mitchell et al ${ }^{29}$. 
SNPs were genotyped using TaqMan probes (Applied Biosystems, Foster City, CA) and the Dynamic Array Integrated Fluidic Circuits (Fluidigm, San Francisco, CA). These genotyping assays included primers to amplify the region containing the SNP of interest and two TaqMan Minor Groove Binder probes that are specific to the polymorphic variant alleles at the site labeled with different fluorescent reporter dyes, FAM and VIC. All reactions were performed using standard conditions supplied by Fluidigm. Following thermocycling, fluorescence levels of the FAM and VIC dyes were measured using the EP1 Reader and genotypes were scored using the Fluidigm Genotyping Analysis software. Genotypes were entered into a laboratory database (Progeny, South Bend, IN) to generate datasets for analysis.

\section{Haplotyping}

Haplotype analysis of SNPs in the same gene or region was used to evaluate regional associations with PPHN. Haplotype analysis using sliding windows of 2-6 SNPs across the region was performed.

\section{Case- Control Study of amino acid levels}

Data on 9 amino acids including arginine and citrulline, measured by tandem mass spectrometry (MS/MS) were obtained from routine newborn screening from State of Iowa Hygienic Laboratory. Analysis included 32 cases and 64 unaffected controls matched for gender, gestational age and year of birth. Forty nine cases were excluded from the original 94 patients studied in the genotyping phase (38 were born prior to August of 2003 before the implementation of MS/MS to the Iowa newborn screening program, 24 were excluded as the initial newborn screening test was obtained either before 24 hours from birth or after 72 hours from birth. Data on analyte measurements were linked to the clinical medical record data.

Approval for use of the newborn screening data was granted by the Iowa Department of Public Health Research and Ethics Review Committee.

\section{Statistical Analysis}

Statistical analyses of the genotype data was performed with a transmission disequilibrium test to look for nonrandom allele transmission from parents to offspring using the PLINK software (http://pngu.mgh.harvard.edu/purcell/plink/). Haplotype analysis was also done using PLINK software. A Bonferroni significance threshold of $\mathrm{P}=0.001$ (0.05/48 markers) was used to correct for multiple testing.

The case-control study analysis was performed with SAS version 9.3 (SAS Institute, Cary, NC). Demographic and clinical characteristics were compared between cohorts using chi square tests for categorical variables and student $t$ tests for continuous variables. A P value of $<0.05$ was considered statistically significant for case control study analysis.

\section{Supplementary Material}

Refer to Web version on PubMed Central for supplementary material. 


\section{Acknowledgments}

We express our sincere thanks to the families that participated in this study. We also thank Nancy Weathers, Allison Momany and Bruce Bedell at the Dagle - Ryckman Laboratory at University of Iowa for their support; Stanton Berberich from the State Hygienic Laboratory and Kimberly Piper from the Congenital and Inherited Disorders Advisory Committee for their assistance in the acquisition of newborn screening data. This work was supported by a K99/R00 grant from the Eunice Kennedy Shriver National Institute of Child Health and Human Development [R00 HD-065786]. The content is solely the responsibility of the authors and does not necessarily represent the official views of the Eunice Kennedy Shriver National Institute of Child Health and Human Development or the National Institutes of Health.

Funding Source: Eunice Kennedy Shriver National Institute of Child Health and Human Development [R00 HD-065786]

\section{References}

1. Walsh-Sukys MC, Tyson JE, Wright LL, et al. Persistent pulmonary hypertension of the newborn in the era before nitric oxide: practice variation and outcomes. Pediatrics. 2000; 105:14-20. [PubMed: 10617698]

2. Nair J, Lakshminrusimha S. Update on PPHN: mechanisms and treatment. Semin Perinatol. 2014; 38(2):78-91. [PubMed: 24580763]

3. The Neonatal Inhaled Nitric Oxide Study Group. Inhaled nitric oxide in full-term and nearly fullterm infants with hypoxic respiratory failure. N Engl J Med. 1997; 336(9):597-604. [PubMed: 9036320]

4. Clark RH, Kueser TJ, Walker MW, et al. Low-dose nitric oxide therapy for persistent pulmonary hypertension of the new- born. N Engl J Med. 2000; 342(7):469-474. [PubMed: 10675427]

5. Abman SH, Chatfield BA, Hall SL, McMurtry IF. Role of endothelium derived relaxing factor during transition of pulmonary circulation at birth. Am J Physiol. 1990; 259:H1921-H1927. [PubMed: 2260716]

6. Fineman JR, Wong J, Morin FC III, Wild LM, Soifer SJ. Chronic nitric oxide inhibition in utero produces persistent pulmonary hypertension in newborn lambs. J Clin Invest. 1994; 93:2675-83. [PubMed: 8201005]

7. Förstermann U, Sessa WC. Nitric oxide synthases: regulation and function. Eur Heart J. 2012; 33(7):829-37. [PubMed: 21890489]

8. Pearson DL, Dawling S, Walsh WF, et al. Neonatal pulmonary hypertension-urea cycle intermediates, nitric oxide production, and carbamoyl-phosphate synthetase function. N Engl J Med. 2001; 344:1832-8. [PubMed: 11407344]

9. Trittmann JK, Nelin LD, Zmuda EJ, et al. Arginase I gene single-nucleotide polymorphism is associated with decreased risk of pulmonary hypertension in bronchopulmonary dysplasia. Acta Paediatr. 2014; 103(10):e439-43. [PubMed: 24919409]

10. Vosatka RJ, Kashyap S, Trifiletti RR. Arginine deficiency accompanies persistent pulmonary hypertension of the newborn. Biol Neonate. 1994; 66(2-3):65-70. [PubMed: 7993949]

11. Hosono S, Ohno T, Kimoto H, et al. Developmental outcomes in persistent pulmonary hypertension treated with nitric oxide therapy. Pediatr Int. 2009; 51(1):79-83. [PubMed: 19371283]

12. Rosenberg AA 1, Lee NR, Vaver KN, et al. School-age outcomes of newborns treated for persistent pulmonary hypertension. J Perinatol. 2010; 30(2):127-34. [PubMed: 19798045]

13. Clark RH, Huckaby JL, Kueser TJ, et al. Clinical Inhaled Nitric Oxide Research Group. Low-dose nitric oxide therapy for persistent pulmonary hypertension: 1-year follow-up. J Perinatol. 2003; 23:300-3. [PubMed: 12774137]

14. Byers HM, Dagle JM, Klein JM, et al. Variations in CRHR1 areassociated with persistent pulmonary hypertension of the newborn. PediatrRes. 2012; 71(2):162-167.

15. Catteruccia M, Verrigni D, Martinelli D, et al. Persistent pulmonary arterial hypertension in the newborn (PPHN): a frequent manifestation of TMEM70 defective patients. Mol Genet Metab. 2014; 111(3):353-9. [PubMed: 24485043] 
16. Vonk JM, Postma DS, Maarsingh H, et al. Arginase 1 and arginase 2 variations associate with asthma, asthma severity and beta2 agonist and steroid response. Genomics. 2010; 20(3):179-86.

17. Litonjua AA 1, Lasky-Su J, Schneiter K, et al. ARG1 is a novel bronchodilator response gene: screening and replication in four asthma cohorts. Am J Respir Crit Care Med. 2008; 178(7):68894. [PubMed: 18617639]

18. Dumont J, Zureik M, Cottel D, et al. Association of arginase 1 gene polymorphisms with the risk of myocardial infarction and common carotid intima media thickness. J Med Genet. 2007; 44(8): 526-31. [PubMed: 17369504]

19. Dumont J 1, Meroufel D, Bauters C, et al. Association of ornithine transcarbamylase gene polymorphisms with hypertension and coronary artery vasomotion. Am J Hypertens. 2009; 22(9): 993-1000. [PubMed: 19574962]

20. Canter JA, Summar ML, Smith HB, et al. Genetic variation in the mitochondrial enzyme carbamylphosphate synthetase I predisposes children to increased pulmonary artery pressure following surgical repair of congenital heart defects: a validated genetic association study. Mitochondrion. 2007; 7(3):204-10. [PubMed: 17188582]

21. Kallianpur AR 1, Hall LD, Yadav M, et al. The hemochromatosis C282Y allele: a risk factor for hepatic veno-occlusive disease after hematopoietic stem cell transplantation. Bone Marrow Transplant. 2005; 35(12):1155-64. [PubMed: 15834437]

22. Summar ML, Hall L, Christman B, et al. Environmentally determined genetic expression: clinical correlates with molecular variants of carbamyl phosphate synthetase I. Mol Genet Metab. 2004; 81(Suppl 1):S12-9. [PubMed: 15050969]

23. Summar ML 1, Hall LD, Eeds AM, et al. Characterization of genomic structure and polymorphisms in the human carbamyl phosphate synthetase I gene. Gene. 2003; 311:51-7. [PubMed: 12853138]

24. Ischiropoulos H, Zhu L, Chen J, et al. Peroxynitrite mediated tyrosine nitration catalyzed by superoxide dismutase. Arch Biochem Biophys. 1992; 298:431-437. [PubMed: 1416974]

25. Halliwell B. What nitrates tyrosine? Is nitrotyrosine specific as a biomarker of peroxynitrite formation in vivo? FEBS Lett. 1997; 411:157-60. [PubMed: 9271196]

26. Banks BA, Ischiropoulos $\mathrm{H}$, McClelland M, et al. Plasma 3-nitrotyrosine is elevated in premature infants who develop bronchopulmonary dysplasia. Pediatrics. 1998; 101:870-4. [PubMed: 9565417]

27. Ryckman KK, Dagle JM, Shchelochkov OA, Ehinger N, et al. Association of amino acids with common complications of prematurity. Pediatr Res. 2013; 73:700-5. [PubMed: 23481552]

28. Ploder M, Neurauter G, Spittler A, Schroecksnadel K, et al. Serum phenylalanine in patients post trauma and with sepsis correlate to neopterin concentrations. Amino Acids. 2008; 35:303-7. [PubMed: 18163176]

29. Mitchell S, Ellingson C, Coyne T, et al. Genetic variation in the urea cycle: a model resource for investigating key candidate genes for common diseases. Hum Mutat. 2009; 30(1):56-60. [PubMed: 18666241] 
Table 1

Demographic and clinical characteristics of study population for genotyping phase

\begin{tabular}{|c|c|}
\hline & Cases $(n=94)$ \\
\hline \multicolumn{2}{|l|}{ Infant Characteristics } \\
\hline Male gender & $61(65)$ \\
\hline Caucasian race & $83(88)$ \\
\hline Birth weight (g) & $3532(+/-626)$ \\
\hline Gestational age (weeks) & $38.3(+/-1.8)$ \\
\hline Postdate (>40 weeks) & $13(17)$ \\
\hline Premature ( $<37$ weeks) & $19(20)$ \\
\hline Apgar $<7$ at $5 \mathrm{~min}$ & $18(19)$ \\
\hline Meconium aspiration & $26(28)$ \\
\hline Pneumothorax & $28(30)$ \\
\hline Pneumonia & $19(20)$ \\
\hline Treated with iNO & $66(70)$ \\
\hline ECMO & $9(10)$ \\
\hline \multicolumn{2}{|l|}{ Maternal Characteristics } \\
\hline Maternal age (years) & $28.7(+/-5.7)$ \\
\hline Cesarean section & $50(53)$ \\
\hline Complications of delivery & $45(48)$ \\
\hline \multicolumn{2}{|l|}{ Cohort descriptors } \\
\hline Triads (infant, mother and father) & $77(82)$ \\
\hline UIHC hospital of birth & $24(26)$ \\
\hline
\end{tabular}

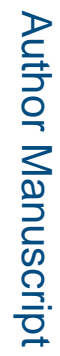

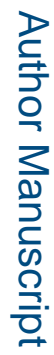

Pediatr Res. Author manuscript; available in PMC 2019 January 01. 


\section{List of Genes and SNPs}

Table 2

\begin{tabular}{|l|l|l|l|}
\hline Gene & Gene name & Chromosome & SNPs \\
\hline NAGS & N-acetylglutamate synthase & 17 & rs228770, rs228771, rs228773, rs186636 \\
\hline CPS1 & Carbamoyl-phosphate synthetase 1 & 2 & $\begin{array}{l}\text { rs16844619, rs17824552, rs13020941, rs6435577, rs4399666, rs2287600, } \\
\text { rs41272667, rs2287599, rs7607412, rs7572146, rs6724941, rs3213784, } \\
\text { rs1047891, rs6748782, rs41272673, rs2216405 }\end{array}$ \\
\hline OTC & Ornithine transcarbamylase & $\mathrm{X}$ & rs5963409, rs5917574, rs5963417, rs5917591, rs5963427 \\
\hline ASS1 & Argininosuccinate synthetase & 9 & $\begin{array}{l}\text { rs11243372, rs7860909, rs1215985, rs11243414, rs10901072, rs2297599, } \\
\text { rs652313, rs540140, rs1215940, rs1215970, rs 12555797, rs10901080, } \\
\text { rs666174, rs634432, rs7018779, rs10114424 }\end{array}$ \\
\hline$A S L$ & Argininosuccinate lyase & 7 & rs160648, rs313829, rs2292938 \\
\hline$A R G 1$ & Arginase 1 & 6 & rs2781667, rs2246012, rs3850245, rs34504481 \\
\hline
\end{tabular}

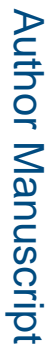

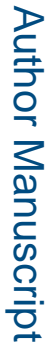

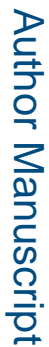

Pediatr Res. Author manuscript; available in PMC 2019 January 01. 
Table 3

\section{Case control analysis of amino acid levels on initial newborn screening}

\begin{tabular}{|c|c|c|c|}
\hline & Cases $(n=32)$ & Controls $(n=64)$ & p value \\
\hline Male Gender * & $20(62.5)$ & $40(62.5)$ & 1.00 \\
\hline Caucasian race ${ }^{*}$ & $29(90.6)$ & $58(90.6)$ & 0.60 \\
\hline Birth weight (g) & $3378(586)$ & $3257(546)$ & 0.32 \\
\hline Gestational age (weeks) & $37.9(1.7)$ & $37.9(1.7)$ & 1.00 \\
\hline Age at initial newborn screening (hours) & $30.9(8.7)$ & $28.3(6.1)$ & 0.14 \\
\hline On TPN at the time of NBS & $2(6.25)$ & $2(3.1)$ & 0.60 \\
\hline Ala & $216.7(106.9)$ & $196.1(74.8)$ & 0.33 \\
\hline $\operatorname{Arg}$ & $4.2(3.3)$ & $4.5(2.4)$ & 0.58 \\
\hline Cit & $9.2(3.8)$ & $9.9(2.6)$ & 0.35 \\
\hline Glu & $148.8(50.8)$ & $148.1(38.5)$ & 0.93 \\
\hline Leu & $108.4(52.6)$ & $100.9(31.6)$ & 0.46 \\
\hline Met & $23.6(14.4)$ & $22.1(7.8)$ & 0.56 \\
\hline Phe & $67.3(21.1)$ & $57.4(12.2)$ & 0.01 \\
\hline Tyr & $53.4(34.1)$ & $78.7(41.8)$ & 0.003 \\
\hline Val & $88.7(39.6)$ & $81.3(21.9)$ & 0.33 \\
\hline
\end{tabular}

Data presented as N (\%). Differences between groups were analyzed by chi-square test.

All other variables presented as Mean (Standard Deviation). Metabolite levels are presented in ( $\mu \mathrm{mol} / \mathrm{L}$ ) units. Differences between groups were analyzed by t-test. 